CPTH-S004.0300

LPTENS 00/07

hep-th/0003050

\title{
RG Flow, Wilson Loops and the Dilaton Tadpole
}

\author{
Carlo Angelantonj $^{\dagger}$ and Adi Armoni ${ }^{\ddagger}$ \\ †Laboratoire de Physique Théorique de l'École Normale Supérieure円 \\ 24, rue Lhomond, F-75231 Paris Cedex 05 \\ angelant@lpt.ens.fr \\ ¥ Centre de Physique Théorique de l'École Polytechniqu母 \\ F-91128 Palaiseau Cedex \\ armoni@cpht.polytechnique.fr
}

\begin{abstract}
We discuss the role of the dilaton tadpole in the holographic description of non-supersymmetric gauge theories that are conformal in the planar limit. On the string theory side, the presence of the dilaton tadpole modifies the AdS background inducing a logarithmic running for the radius and the dilaton. Using the holographic prescription we compute the Wilson loop on the gravity side and find a smooth interpolating potential between asymptotic freedom and confinement, as expected from field theory.
\end{abstract}

\footnotetext{
${ }^{1}$ Unité mixte du CNRS et de l'ENS, UMR 8549

${ }^{2}$ Unité mixte du CNRS et de l'EP, UMR 7644
} 


\section{Introduction}

The AdS/CFT correspondence has improved considerably our understanding of strongly coupled large $N$ gauge theories [1], showing that the natural description of $4 \mathrm{~d}$ theories in this regime is in terms of $5 \mathrm{~d}$ gravity, where the extra dimension is related to the $4 \mathrm{~d}$ energy scale. So far, strong evidence for the validity of the conjecture has come from the description of $\mathcal{N}=4 \mathrm{SYM}$ in terms of IIB supergravity, while limited efforts have been devoted to the holographic description of non-supersymmetric non-conformal field theories. Moreover, the few examples analyzed were mostly based on the tachyonic (oriented) OB string [2, 3], where the presence of the tachyon complicates the gravity description [4, 5] and leads to instabilities in the dual strongly coupled field theory [6].

The first attempt to find a non-tachyonic holographic description of non-supersymmetric gauge theories was recently made in [7]. The analysis was still performed within the framework of $0 \mathrm{~B}$ strings, but resorting to a particular orientifold projection [8] to eliminate the tachyon from the bulk and to avoid problems with the doubling of R-R sectors. Actually, the gauge theories that naturally emerge from (orientifolds of) type 0 models have the peculiar property of becoming conformal in the planar limit, and therefore several results can be mutuated from $\mathcal{N}=4$ [9, 7]. In particular, the leading (planar) geometry is known to be of the form $A d S_{5} \times X_{5}$ with a constant dilaton field. For finite $N$, however, the gauge theory is no longer conformal and new features are expected in the gravity description. Indeed, a general property of non-supersymmetric theories seems to be the presence of a non-vanishing dilaton tadpole at genus one-half [10]. On the other hand, supersymmetry relates R-R tadpoles to NS-NS ones, and thus demanding neutral configurations of orientifold planes and D-branes automatically ensures the vanishing of all massless tadpoles. However, whenever supersymmetry is not present one can find configurations for which the R-R and NS-NS tadpole conditions are incompatible [8, 10, 11]. While uncanceled R-R tadpoles manifest themselves in pathologies of the string theory [12], NS-NS tadpoles are less problematic. They correspond to potential terms in the low-energy action and their main effect is to modify the vacuum. In our case this is precisely what one needs! Indeed, studying D3 branes in non-tachyonic orientifolds of type 0B (with $\mathrm{O}^{\prime} 7$-planes and D7-branes, where a prime indicates the non-tachyonic involution) we showed explicitly [7] how a genus one-half dilaton tadpole in the bulk theory does reproduce qualitatively and quantitatively the expected logarithmic gauge theory RG flow. (Recently, a logarithmic running of the gauge coupling was also found in the gravitational description of

$\mathcal{N}=2 \mathrm{SU}(N) \times \mathrm{SU}(N+M)$ gauge theory 13 . See related discussion on dilaton-induced $\mathrm{RG}$ flow in [14].)

The purpose of this letter is to pursue this program further, studying the back-reaction on the metric induced by the presence of a dilaton tadpole. In particular, we compute the 
Wilson loop in string theory and find a quark anti-quark potential interpolating between a (logarithmically running) Coulomb phase and a confining phase in the IR, as expected from the field theory analysis. To the best of our knowledge, the interpolating solution that we find is the only known example in which a single solution describes the expected behavior in the entire energy regime. Although our analysis is quite general and applies to a vast class of non-supersymmetric non-conformal theories for which a non-vanishing dilaton tadpole is expected to develop [10], the simplest model one may conceive originates from D3-branes and $\mathrm{O}^{\prime} 3$-planes in type 0B. In the large $N$ limit the near horizon geometry is $A d S_{5} \times \mathbb{R} \mathbb{P}^{5}[15]$, where $\mathbb{R} \mathbb{P}^{5}=S^{5} / \mathbb{Z}_{2}$, with $\mathbb{Z}_{2}$ the non-tachyonic involution [8] associated to $\mathrm{O}^{\prime} 3$. Since the $\mathbb{Z}_{2}$ acts freely on $S^{5}$, no open sector is present in the bulk and the spectrum of the model consists of all the $\mathbb{Z}_{2}$-invariant harmonics of the $0 \mathrm{~B}$ fields. In particular, the tachyon is projected out. Moreover, since two-cycles of $\mathbb{R P}^{5}$ are unorientable, as for instance $\mathbb{R P}^{2} \subset \mathbb{R P}^{5}$, new interactions in the low-energy action are expected to originate from unoriented world-sheet topologies. These are weighted by generic (not only even) powers of the dilaton, and in particular a dilaton tadpole can be generated. Unfortunately, an explicit computation of one-point amplitudes for the dilaton in the curved $A d S_{5} \times \mathbb{R} \mathbb{P}^{5}$ geometry seems out of reach within the actual (perturbative) definition of string theory, although such a term is expected from the above arguments, from our experience with non-supersymmetric theories, and from the results we are going to present here.

The organization of this letter is as follows: in section 2 we introduce the class of field theories we want to study, and present an explicit example from (unoriented) D-branes in the $0 \mathrm{~B}$ string. In section 3 we turn to the gravity description and find an explicit solution for the dilaton and the metric up to next-to-leading order in $1 / N$ expansion. In section 4 we extract the holographic quark anti-quark potential and compare it with the field theory analysis of section 2. Finally, section 5 contains our final comments about the validity of the gravity description.

\section{Field theory considerations}

One of the most remarkable results in field theory is the running of coupling constants

$$
\frac{d \alpha(u)}{d \log u}=-\beta(\alpha)
$$

where $\alpha(u)=g_{\mathrm{YM}}^{2}(u) N$ is the 't Hooft coupling. The precise expression of the betafunction is model dependent and encodes the quantum corrections to the classical behavior

$\alpha(u)=\alpha_{0}$. Whenever a quantum theory does not depend on any scale and thus is exactly conformally invariant, as for instance $\mathcal{N}=4 \mathrm{SYM}, \beta(\alpha)$ vanishes identically and the 
coupling constants take their classical values for all energy scales. Combining these two arguments, it is then evident that theories that are conformally invariant in the planar limit are governed by a RG equation

$$
\frac{d \alpha(u)}{d \log u}=-\frac{1}{N} \beta_{1}(\alpha)-\frac{1}{N^{2}} \beta_{2}(\alpha)+\ldots
$$

so that one recovers the expected $\alpha(u)=\alpha_{0}$ result for $N \rightarrow \infty$. Here, $\beta_{i}(\alpha)$ do not represent the $i^{\text {th }}$-loop contribution to the RG equation. Rather, they encode all the contributions at a given order in $1 / N$.

To be concrete, the simplest prototype one can conceive is an $\mathrm{SU}(N)$ gauge theory with six real scalars in the adjoint and 4 Weyl spinors in the antisymmetric and antisymmetricconjugate representations, arising from the non-tachyonic orientifold projection on type 0B D3 branes [8, 11]. This theory was shown to converge to the $\mathrm{SU}(N) \mathcal{N}=4 \mathrm{SYM}$ in the planar limit [0], and to have a one-loop beta-function suppressed by $1 / N$ (with $b_{1}=\frac{16}{3}$ ), and a two-loop beta-function contributing both to $\beta_{1}(\alpha)$ and $\beta_{2}(\alpha)$ in (2) [3].

One can extract some interesting results from the RG equation (2). Since in (2) there is a natural small parameter, $1 / N$, it is natural to solve this equation by iterations

$$
\alpha(u)=\alpha_{0}+\frac{1}{N} \alpha_{1}+\ldots .
$$

The solution

$$
\alpha(u)=\alpha_{0}-\frac{1}{N} \beta_{1}\left(\alpha_{0}\right) \log u
$$

that, to this order, can be equivalently written as

$$
\frac{1}{\alpha(u)}=\frac{1}{\alpha_{0}}+\frac{1}{N} \frac{\beta_{1}\left(\alpha_{0}\right)}{\alpha_{0}^{2}} \log u
$$

leads then to a quark anti-quark potential

$$
E(L)=\frac{\alpha(L)}{L}=\frac{\alpha_{0}}{L\left(1-\frac{1}{N} \frac{\beta_{1}\left(\alpha_{0}\right)}{\alpha_{0}} \log L\right)},
$$

as expected for asymptotically free theories. Actually, since for large $N$ the beta-function varies slowly, the logarithmic behavior (5) persists beyond the perturbative regime, up to energy scales such that $\left|\frac{1}{N} \frac{\beta_{1}\left(\alpha_{0}\right)}{\alpha_{0}^{2}} \log u\right| \ll 1$. Note that the expansion is not valid in the (extreme) IR.

The description of the (non-perturbative) IR regime is less straightforward. Although for the specific model we introduced previously the scalars do not acquire a potential at the one-loop level [3, 7], in general one would not expect to find a moduli space of vacua for non-supersymmetric theories. Thus, a potential for the scalars should develop: the scalars might become massive, or tachyonic, and might also acquire a non-vanishing vacuum 
expectation value. Although the specific shape of the potential is model dependent, in the following we shall assume that the scalars become massive without acquiring any vev. As we shall see, the string theory description supports this assumption.

Once the massive scalars decouple, the IR degrees of freedom will consist of YangMills theory with four massless spinors (a QCD like theory), and a confining potential is expected

$$
E(L)=\sigma L
$$

where $\sigma=\Lambda_{\mathrm{QCD}}^{2}$ is the QCD string tension. For pure (large $N$ ) Yang-Mills theory one expects a constant (finite) string tension $\sigma_{0}$. In the present case, however, one expects a different behavior. Since the theories we are describing are conformal in the planar limit, the string tension should to vanish accordingly. To estimate the $N$ dependence of the string tension, one can integrate the RG equation (2) from an arbitrary weak coupling scale $\left(\sim u_{0}\right.$ in the UV) to a strong coupling one $\left(\sim \Lambda_{\mathrm{QCD}}\right)$ :

$$
\Lambda_{\mathrm{QCD}}=u_{0} e^{-N \int \frac{d \alpha}{\beta_{1}(\alpha)}}
$$

that translates into an exponentially suppressed string tension

$$
\sigma \sim e^{-N}
$$

\section{The gravity solution}

We now turn to a dual gravitational description of the gauge theories introduced in the previous section, in the spirit of the AdS/CFT correspondence [1]. As discussed in [7], the dual gravity description involves the ten-dimensional metric, the dilaton and the R-R four-form potential (with self-dual field strength), whose dynamics is essentially encoded in the action'

$$
S=\frac{1}{\left(\alpha^{\prime}\right)^{4}} \int d^{10} x \sqrt{-g}\left[e^{-2 \Phi}\left(R+4 \partial_{\rho} \Phi \partial^{\rho} \Phi\right)+\frac{1}{\alpha^{\prime}} C e^{-\Phi}-\frac{1}{4}\left(\alpha^{\prime}\right)^{4}\left|F_{5}\right|^{2}\right],
$$

or in its equations of motion

$$
\begin{aligned}
e^{-2 \Phi}\left(8(\nabla \Phi)^{2}-8 \nabla^{2} \Phi-2 R\right)-C e^{-\Phi} & =0 \\
e^{-2 \Phi}\left(R_{\mu \nu}+2 \nabla_{\mu} \nabla_{\nu} \Phi\right)+\frac{1}{4} g_{\mu \nu} C e^{-\Phi} & \\
-\frac{1}{96}\left(F_{\mu \rho \sigma \lambda \tau} F_{\nu}{ }^{\rho \sigma \lambda \tau}-\frac{1}{10} g_{\mu \nu} F_{\rho \sigma \lambda \tau \eta} F^{\rho \sigma \lambda \tau \eta}\right) & =0 .
\end{aligned}
$$

The term $C e^{-\Phi}=C g_{s}^{-1}$ corresponds to the dilaton tadpole expected from half-genus in string perturbation theory. Although the direct computation of this term is difficult, there are plausible arguments in favor of its presence, as discussed in the introduction.

\footnotetext{
${ }^{3}$ To be more precise one should use the PST action [16], since we are dealing with a self-dual 5 -form $F_{5}$.
} 
In the spirit of AdS/CFT correspondence, we make for the metric the ansatz

$$
d s^{2}=\alpha^{\prime}\left(d \tau^{2}+e^{2 \lambda(\tau)} \eta^{a b} d x_{a} d x_{b}+e^{2 \nu(\tau)} d \Omega_{5}^{2}\right),
$$

compatible with four-dimensional Poincaré invariance and $\mathrm{SU}(4)$ flavor symmetry. Moreover, the five-form field strength has $N-\frac{1}{2}$ units of flux induced by the $N$ D3 branes and the $\mathrm{O}^{\prime} 3$ plane. In the following we shall ignore the contribution of the orientifold plane, manifestly suppressed in the presence of a large number of branes.

Inserting the above ansatz in the eqs. (11) and (12) and allowing for a $\tau$ dependence of the various fields one obtains

$$
\begin{aligned}
2 \ddot{\Phi}-4 \ddot{\lambda}-5 \ddot{\nu}-4 \dot{\lambda}^{2}-5 \dot{\nu}^{2}+\frac{1}{2} N^{2} e^{2 \Phi-10 \nu}-\frac{1}{4} C e^{\Phi} & =0, \\
\ddot{\lambda}-(2 \dot{\Phi}-4 \dot{\lambda}-5 \dot{\nu}) \dot{\lambda}-\frac{1}{2} N^{2} e^{2 \Phi-10 \nu}+\frac{1}{4} C e^{\Phi} & =0, \\
\ddot{\nu}-(2 \dot{\Phi}-4 \dot{\lambda}-5 \dot{\nu}) \dot{\nu}-4 e^{-2 \nu}+\frac{1}{2} N^{2} e^{2 \Phi-10 \nu}+\frac{1}{4} C e^{\Phi} & =0, \\
-12 \dot{\lambda}^{2}-20 \dot{\nu}^{2}-4 \dot{\Phi}^{2}+20 \dot{\Phi} \dot{\nu}+16 \dot{\Phi} \dot{\lambda}-40 \dot{\lambda} \dot{\nu} & \\
+20 e^{-2 \nu}-N^{2} e^{2 \Phi-10 \nu}+C e^{\Phi} & =0 .
\end{aligned}
$$

As usual in General Relativity, the $\tau \tau$ component (17) of Einstein's equations is not independent, and translates into a vanishing energy constraint for the associated mechanical system.

In order to better appreciate the role of the different contributions in the low-energy action, it is useful to redefine $\Phi \rightarrow \Phi-\log N$, in order that $e^{\Phi}$ be directly related to the 't Hooft coupling. The (independent) field equations then read

$$
\begin{aligned}
2 \ddot{\Phi}-4 \dot{\Phi}^{2}+8 \dot{\Phi} \dot{\lambda}+10 \dot{\Phi} \dot{\nu}+3 \frac{C}{N} e^{\Phi} & =0, \\
\ddot{\lambda}-(2 \dot{\Phi}-4 \dot{\lambda}-5 \dot{\nu}) \dot{\lambda}-\frac{1}{2} e^{2 \Phi-10 \nu}+\frac{1}{4} \frac{C}{N} e^{\Phi} & =0, \\
\ddot{\nu}-(2 \dot{\Phi}-4 \dot{\lambda}-5 \dot{\nu}) \dot{\nu}-4 e^{-2 \nu}+\frac{1}{2} e^{2 \Phi-10 \nu}+\frac{1}{4} \frac{C}{N} e^{\Phi} & =0 .
\end{aligned}
$$

From these one can neatly understand the role of the dilaton tadpole: it represents a $1 / N$ correction to the leading type IIB supergravity equations, and thus induces $1 / N$ corrections to the $A d S_{5} \times \mathbb{R P}^{5}$ leading geometry, in agreement with field theory expectations. Moreover, $1 / N$ is a natural small parameter in the theory and suggests to solve the equations by iterations

$$
\begin{aligned}
\Phi(\tau) & =\Phi_{0}(\tau)+\frac{1}{N} \Phi_{1}(\tau)+\ldots \\
\lambda(\tau) & =\lambda_{0}(\tau)+\frac{1}{N} \lambda_{1}(\tau)+\ldots \\
\nu(\tau) & =\nu_{0}(\tau)+\frac{1}{N} \nu_{1}(\tau)+\ldots
\end{aligned}
$$


The leading order equations

$$
\begin{aligned}
2 \ddot{\Phi}_{0}-4 \dot{\Phi}_{0}^{2}+8 \dot{\Phi}_{0} \dot{\lambda}_{0}+10 \dot{\Phi}_{0} \dot{\nu}_{0} & =0, \\
\ddot{\lambda}_{0}-\left(2 \dot{\Phi}_{0}-4 \dot{\lambda}_{0}-5 \dot{\nu}_{0}\right) \dot{\lambda}_{0}-\frac{1}{2} e^{2 \Phi_{0}-10 \nu_{0}} & =0, \\
\ddot{\nu}_{0}-\left(2 \dot{\Phi}_{0}-4 \dot{\lambda}_{0}-5 \dot{\nu}_{0}\right) \dot{\nu}_{0}-4 e^{-2 \nu_{0}}+\frac{1}{2} e^{2 \Phi_{0}-10 \nu_{0}} & =0,
\end{aligned}
$$

independent of the dilaton tadpole, are consistently solved by the expected (leading) $A d S_{5} \times \mathbb{R P}^{5}$ geometry

$$
\begin{aligned}
& \Phi_{0}=\varphi, \\
& \lambda_{0}=\sqrt[8]{8} e^{-\frac{1}{4} \varphi} \tau, \\
& \nu_{0}=-\frac{1}{8} \log 8+\frac{1}{4} \varphi .
\end{aligned}
$$

The next-to-leading order equations then reduce to

$$
\begin{aligned}
\ddot{\Phi}_{1}+4 \sqrt[8]{8} e^{-\frac{1}{4} \varphi} \dot{\Phi}_{1}+\frac{3}{2} C e^{\varphi} & =0, \\
\ddot{\lambda}_{1}+\sqrt[8]{8} e^{-\frac{1}{4} \varphi}\left(-2 \dot{\Phi}_{1}+8 \dot{\lambda}_{1}+5 \dot{\nu}_{1}\right)-8 \sqrt[4]{8} e^{-\frac{1}{2} \varphi}\left(\Phi_{1}-5 \nu_{1}\right)+\frac{1}{4} C e^{\varphi} & =0, \\
\ddot{\nu}_{1}+4 \sqrt[8]{8} e^{-\frac{1}{4} \varphi} \dot{\nu}_{1}+8 \sqrt[8]{8} e^{-\frac{1}{2} \varphi}\left(\Phi_{1}-5 \nu_{1}\right)+\frac{1}{4} C e^{\varphi} & =0,
\end{aligned}
$$

and are solved by

$$
\begin{aligned}
& \Phi_{1}=-\frac{3}{8 \sqrt[8]{8}} C e^{\frac{5}{4} \varphi} \tau, \\
& \lambda_{1}=\frac{3}{64} C e^{\varphi} \tau^{2}-\frac{15}{256 \sqrt[8]{8}} C e^{\frac{5}{4} \varphi} \tau, \\
& \nu_{1}=-\frac{3}{32 \sqrt[8]{8}} C e^{\frac{5}{4} \varphi} \tau-\frac{1}{32} 8^{-\frac{5}{4}} C e^{\frac{3}{2} \varphi} .
\end{aligned}
$$

In principle one should also consider the higher order iterations. However, we shall stop here since in the low energy action we are already neglecting contributions of higher order in $1 / N$, as for example a cosmological constant.

Collecting the various contributions, one finds the following expressions for the dilaton

$$
\Phi=\varphi-\frac{3}{8} \frac{1}{\sqrt[8]{8}} \frac{C}{N} e^{\frac{5}{4} \varphi} \tau,
$$

and for the metric tensor

$$
\begin{aligned}
\frac{1}{\alpha^{\prime}} d s^{2}= & d \tau^{2}+\exp \left[2 \sqrt[8]{8} e^{-\frac{1}{4} \varphi} \tau+\frac{3}{32} \frac{C}{N} e^{\varphi} \tau^{2}\right] \eta_{a b} d x^{a} d x^{b} \\
& +\exp \left[-\frac{1}{4} \log 8+\frac{1}{2} \varphi-\frac{3}{16 \sqrt[8]{8}} \frac{C}{N} e^{\frac{5}{4} \varphi} \tau\right] d \Omega_{5}^{2},
\end{aligned}
$$

It is not hard to realize that the introduction of a dilaton tadpole results into a running dilaton and running radii for the $A d S_{5}$ and $\mathbb{R P}^{5}$ spaces. This can be better appreciated if one parametrizes the metric as

$$
\frac{1}{\alpha^{\prime}} d s^{2}=R^{2}(u) \frac{d u^{2}}{u^{2}}+\frac{u^{2}}{R^{2}(u)} \eta_{a b} d x^{a} d x^{b}+\tilde{R}^{2}(u) d \Omega_{5}^{2},
$$


in terms of the energy scale

$$
u=\int e^{\lambda(\tau)} d \tau
$$

with the radii

$$
R(u)=\frac{d \tau}{d \log u}, \quad \tilde{R}(u)=e^{\nu(\tau(u))} .
$$

Collecting our previous results, we find

$$
\begin{aligned}
\tau & =\frac{1}{\sqrt[8]{8}} e^{\frac{1}{4} \varphi}\left(\log u-\frac{3}{64} \frac{C}{N} e^{\varphi} \log ^{2} u\right) \\
R^{2}(u) & =\frac{\frac{1}{\sqrt[4]{8}} e^{\frac{1}{2} \varphi}}{1+\frac{3}{16} \frac{C}{N} e^{\varphi} \log u} \\
\tilde{R}^{2}(u) & =\frac{\frac{1}{\sqrt[4]{8}} e^{\frac{1}{2} \varphi}}{1+\frac{3}{16 \sqrt[4]{8}} \frac{C}{N} e^{2 \varphi} \log u}, \\
e^{-\Phi} & =e^{-\varphi}+\frac{3}{8 \sqrt[4]{8}} \frac{C}{N} e^{\varphi} \log u .
\end{aligned}
$$

At this point, before we interpret these results, various comments are in order. First, in the above expressions we are repeatedly ignoring $1 / N^{2}$ sub-leading terms. Moreover, the value of $e^{\varphi}$ can not be interpreted any longer as the 't Hooft coupling, since now the field theory is not conformal and the gauge coupling runs. In addition, from eqs. (42) and (43) one might argue that $R$ and $\tilde{R}$ develop singularities at some point in the IR (notice that for our model $C$ is positive and then singularity presents itself for $\log u<0$ ). This, however, is not the case. The singularities are at points beyond the validity of the $1 / N$ approximation and are an artifact of our choice of coordinates. Note, in fact, that the metric (37) is well defined for every energy scale. Finally, contrary to the familiar $\mathcal{N}=4$ super-conformal case the radii $R$ and $\tilde{R}$ have now different values. This is expected since at order $1 / N$ the dilaton tadpole behaves like an effective cosmological constant, and therefore (at that order) the overall curvature of the ten-dimensional space is no longer vanishing.

We can now turn to the interpretation of eqs. (42) and (44), in the spirit of AdS/CFT correspondence. The behavior of the dilaton is consistent with the fact that, in the dual gauge theory, no longer conformal, the coupling constant is expected to run. More precisely, once the dilaton is identified with the 't Hooft coupling, $e^{\Phi(u)} \sim \alpha(u)$, eq. (44) reproduces precisely the logarithmic behavior (5) expected from the gauge theory side, as already shown in [7]. Moreover, in the large $N$ limit the running of the coupling constant is suppressed, in agreement with the fact that in this limit the theory is conformal. Following [7], it would be interesting to compute also in this case the numerical value of the dilaton tadpole $C$ and compare it with the one-loop beta-function coefficient $b_{1}=\frac{16}{3}$. To this end, however, one should compute the contributions from closed strings in the $A d S_{5} \times \mathbb{R} \mathbb{P}^{5}$ 
background with unoriented world-sheet wrapping the $\mathbb{R P}^{5}$. Unfortunately, computations in genuinely curved backgrounds are still an open problem in string perturbation theory.

Following [18 the running of the AdS radius plays a natural role in the computation of the quark anti-quark potential, the subject of the next section.

\section{The Wilson loop}

We can now use the "running" $A d S_{5} \times \mathbb{R P}^{5}$ geometry to compute the quark anti-quark potential using the holographic Wilson loop (see [17] for a recent review). In [19, 18] it was suggested that a natural description of the Wilson loop in terms of gravity is

$$
\langle W(\mathcal{C})\rangle \sim e^{-S}
$$

where, in the limit of large 't Hooft coupling, $S$ is the proper area of a string world-sheet

$$
S=\frac{1}{2 \pi \alpha^{\prime}} \int d \tau d \sigma \sqrt{\operatorname{det} g_{\mu \nu} \partial_{\alpha} X^{\mu} \partial_{\beta} X^{\nu}}
$$

describing the loop $\mathcal{C}$ on the boundary of AdS. The quark anti-quark potential can then be extracted choosing the standard rectangular loop with sides $L$ and $T$, with $T \rightarrow \infty$.

Using the parametrization (38) for the metric $g_{\mu \nu}$, the Nambu-Goto action reads

$$
S / T=E=\int d x \sqrt{\left(\partial_{x} u\right)^{2}+u^{4} / R^{4}(u)},
$$

and thus the quark anti-quark separation and the interaction energy are 18

$$
L \sim \frac{R_{0}^{2}}{u_{0}} \int \frac{d y}{y^{2} \sqrt{y^{4}-R_{0}^{4} / R^{4}\left(y u_{0}\right)}}
$$

and

$$
E \sim u_{0} \int \frac{y^{2} d y}{\sqrt{y^{4}-R_{0}^{4} / R^{4}\left(y u_{0}\right)}}
$$

with $R_{0}=R\left(u_{0}\right)$.

If the energy $u_{0}$ is within the range of validity of the expansion, $\frac{1}{N} \log u_{0} \ll 1$, the ratio $R_{0}^{4} / R^{4}\left(y u_{0}\right)$ can be approximated by

$$
\frac{R_{0}^{4}}{R^{4}\left(y u_{0}\right)} \sim \frac{1+\frac{3}{8} \frac{C}{N} e^{\varphi} \log y u_{0}}{1+\frac{3}{8} \frac{C}{N} e^{\varphi} \log u_{0}} \sim 1+\frac{3}{8} \frac{C}{N} e^{\varphi} \log y .
$$

Thus, one has

$$
L \sim \frac{R_{0}^{2}}{u_{0}}, \quad \text { and } \quad E \sim u_{0}
$$


and the potential

$$
E \sim \frac{R_{0}^{2}}{L} \sim \frac{R^{2}(1 / L)}{L}=\frac{\frac{1}{\sqrt[4]{8}} e^{\frac{1}{2} \varphi}}{L\left(1-\frac{3}{16} \frac{C}{N} e^{\varphi} \log L\right)}
$$

can be expressed in terms of the running AdS radius, similarly to [4].

Eq. (52) reproduces precisely the behavior expected from the gauge theory side: at finite $N$ the Coulomb phase is characterized by a quark anti-quark potential (6) with a (logarithmically) running gauge coupling. The fact that the two prescriptions, the dilaton running

$$
E=\frac{\alpha(L)}{L}=\frac{e^{\Phi(L)}}{L} \sim \frac{e^{\varphi}}{L\left(1-\frac{3}{8 \sqrt[4]{8}} \frac{C}{N} e^{2 \varphi} \log L\right)}
$$

and the Wilson loop (52), yield similar results (in contrast to tachyonic type 0 models [5, (4) is encouraging, although different powers of $e^{\varphi}$ are still involved in the two cases. Note that (53) is a perturbative prescription in contrast to (52) that should, a priori, be applicable to the non-perturbative regime.

Finally, let us describe the IR behavior of the gauge theory. To this end, it is simpler to work with the metric in the form (37). The quark anti-quark energy is then

$$
E=\int d x \sqrt{e^{\lambda(\tau)}\left(\partial_{x} \tau\right)^{2}+e^{2 \lambda(\tau)}}
$$

and, since

$$
\lambda(\tau)=\alpha \tau+\frac{1}{N} \beta \tau^{2}
$$

with $\alpha$ and $\beta$ positive constants, $e^{\lambda}$ has a minimum at $\tau_{\min }=-\alpha N / 2 \beta$, that corresponds to the IR, implying a confining long distance potential 20]

$$
E \sim e^{\lambda_{\min }} L=\sigma L
$$

The reason behind this result is rather simple to understand. For large enough separation, the string will prefer to stay at the minimum of $\lambda$, thus minimizing the action (54). Therefore the energy will be simply proportional to the separation on the AdS boundary. Moreover, the QCD string tension (measured in units of $1 / \alpha^{\prime}$ ) 20]

$$
\sigma=\exp \lambda_{\min }=\exp \left(-\frac{\alpha^{2} N}{4 \beta}\right)=\exp \left(-\frac{16}{3} \sqrt[4]{8} e^{-\frac{3}{2} \varphi} C^{-1} N\right)
$$

is exponentially suppressed with $N$, in agreement with field theory analysis (9).

\section{Comments}

In conclusion, let us make a few comments about the validity of the holographic description that we have just presented. The holographic conjecture relates $4 \mathrm{~d}$ strongly 
coupled gauge theories to $5 \mathrm{~d}$ (super)gravity, and is thus valid, in principle, only within a certain range of energies. Moreover, the procedure of solving the gravitational equations by iterations further restricts the solution. First, we are taking into account only the next-to-leading contribution associated to the dilaton tadpole (a $1 / N$ effect), and therefore we need $N \gg 1$ to be sure that higher order string loops be negligible. Moreover, the procedure of solving the differential equations by iterations is valid only when the expansion parameter is small. Since the expansion parameter is $\epsilon \equiv \frac{1}{N} e^{\frac{3}{2} \varphi} \log u$, the solution is valid only in some regions of the $(u, N)$ space. Note, however, that for any given energy $u$ and initial AdS radius $e^{\frac{1}{4} \varphi}$, it is always possible to find an $N$ such that $\epsilon$ is small, compatibly with the previous requirement. Finally, the curvature of the space should be small enough to allow one to trust classical gravity and to neglect $\alpha^{\prime}$ corrections. Once the previous requirements are met, this last constraint simply translates into large $e^{\frac{1}{4} \varphi}$, as in the $\mathcal{N}=4$ case.

Let us now see whether our solution meets these requirements. It is not hard to see that large $e^{\varphi}$ and small expansion parameter $\epsilon$ translate into large 't Hooft coupling. But, from the field theory considerations, one expects the logarithmic behavior for small and intermediate coupling (5). One should assume non-large $e^{\varphi}$ and therefore $\alpha^{\prime}$ corrections should not be neglected, thus invalidating our solution. However, this is not the case. A more careful analysis reveals that our results depend only on the existence of an $A d S_{5} \times$ $\mathbb{R P}^{5}$ solution at infinite $N$. Now, since it is a common lore that $\alpha^{\prime}$ corrections do not change the geometry of the space [21] (namely, that $\mathcal{N}=4$ is described by an $A d S_{5} \times S^{5}$ metric at any coupling), the logarithmic running should not be affected. Moreover, there seem to be problems in the computation of the short-distance Wilson loop. First, the direct Wilson loop computation results into a value for the one-loop beta-function different from that obtained by the running dilaton recipe. This is actually related to $\alpha^{\prime}$ corrections that, as in the $\mathcal{N}=4$ case [21], are expected to affect the parameters, but not the qualitative picture. In addition, the string world-sheet "invades" also regions where $\epsilon$ is not small. Namely, the holographic computation of the Wilson loop for a given quark anti-quark separation $L$ requires that the metric be known everywhere throughout the range $(u \sim 1 / L, \infty)$. In order to avoid this problem and thus be able to trust our solution, the world-sheet should extend deeply inside AdS, and thus the potential (52) applies only to not too small quark anti-quark separations (i.e. our results are not valid in the extreme UV). Finally, the confining phase for long distances relies on the existence of a minimum for $\lambda(\tau)$. However, at $\tau=\tau_{\min }$ the contribution of next-to-leading term $\lambda_{1}$ is of the same order as $\lambda_{0}$. Thus, we can not exclude that higher order contributions could eliminate the minimum, but hopefully the only effect of higher corrections could be simply to shift $\tau_{\text {min. }}$. However, a definite answer to this problem requires an exact solution to the exact low-energy action. 
Acknowledgments. We are grateful to E. Gardi, A. Sagnotti, D. Seminara and especially to G. Grunberg and J. Sonnenschein for useful comments. A.A. thanks the Department of Applied Mathematics and Theoretical Physics at Cambridge University for the warm hospitality while this work was being completed. This research was supported in part by EEC under TMR contract ERBFMRX-CT96-0090.

\section{References}

[1] J. M. Maldacena, Adv. Theor. Math. Phys. 2 (1998) 231; S. S. Gubser, I. R. Klebanov and A. M. Polyakov, Phys. Lett. B428 (1998) 105; E. Witten, Adv. Theor. Math. Phys. 2 (1998) 253. See also O. Aharony, S. S. Gubser, J. Maldacena, H. Ooguri and Y. Oz, hep-th/9905111 for a recent review.

[2] I.R. Klebanov and A.A. Tseytlin, Nucl. Phys. B546 (1999) 155; JHEP 9903 (1999) 015; M. Billó, B. Craps and F. Roose, Phys. Lett. B457 (1999) 61; A. Armoni and B. Kol, JHEP 9907 (1999) 011.

[3] R. Blumenhagen, A. Font and D. Lüst, Nucl. Phys. B560 (1999) 66.

[4] J. A. Minahan, JHEP 9904 (1999) 007.

[5] J.A. Minahan, JHEP 9901 (1999) 020; I.R. Klebanov and A.A. Tseytlin, Nucl. Phys. B547 (1999) 143; A. Armoni, E. Fuchs and J. Sonnenschein, JHEP 9906 (1999) 027; M. Alishahiha, A. Brandhuber and Y. Oz, JHEP 9905 (1999) 024.

[6] I. R. Klebanov, Phys. Lett. B466 (1999) 166.

[7] C. Angelantonj and A. Armoni, hep-th/9912257, to appear in Nucl. Phys. B.

[8] A. Sagnotti, hep-th/9509080, hep-th/9702093.

[9] S. Kachru and E. Silverstein, Phys. Rev. Lett. 80 (1998) 4855; Z. Kakushadze, Nucl. Phys. B529 (1998) 157.

[10] C. Angelantonj, Phys. Lett. B444 (1998) 309; R. Blumenhagen and A. Kumar, Phys. Lett. B464 (1999) 46; S. Sugimoto, Prog.Theor.Phys. 102 (1999) 685; I. Antoniadis, E. Dudas and A. Sagnotti, Phys. Lett. B464 (1999) 38; G. Aldazabal and A. M. Uranga, JHEP 9910 (1999) 024; K. Förger, Phys. Lett. B469 (1999) 113; C. Angelantonj, I. Antoniadis, G. D'Appollonio, E. Dudas and A. Sagnotti, hep-th/9911081; M. Bianchi, J.F. Morales and G. Pradisi, hep-th/9910228; A. M. Uranga, hepth/9912145.

[11] R. Blumenhagen, A. Font and D. Lüst, Nucl. Phys. B558 (1999) 159. 
[12] J. Polchinski and Y.C. Cai, Nucl. Phys. B296 (1988) 91; M. Bianchi and F. Morales, hep-th/0002149.

[13] I. R. Klebanov and N. A. Nekrasov, hep-th/9911096; I. R. Klebanov and A. A. Tseytlin, hep-th/0002159.

[14] E. Alvarez and C. Gomez, Nucl.Phys. B566 (2000) 363-372; J. de Boer, E. Verlinde and H. Verlinde, hep-th/9912012; E. Verlinde and H. Verlinde, hep-th/9912018; E. Alvarez and C. Gomez, hep-th/0001016.

[15] E. Witten, JHEP 07 (1998) 006.

[16] P. Pasti, D. Sorokin and M. Tonin, Phys. Rev. D55 (1997) 6292.

[17] J. Sonnenschein, hep-th/0003032.

[18] J. M. Maldacena, Phys. Rev. Lett. 80 (1998) 4859.

[19] S.-J. Rey and J. Yee, hep-th/9803001.

[20] Y. Kinar, E. Schreiber and J. Sonnenschein, Nucl. Phys. B566 (2000) 103.

[21] T. Banks and M.B. Green, JHEP 9805 (1998) 002; R. Kallosh, A. Rajaraman, Phys.Rev. D58 (1998) 125003. 